\title{
7 Exposure Assessment for Pesticides in Epidemiological Studies
}

\author{
DICK HEEDERIK ${ }^{\mathbf{1}}$ and KAY TESCHKE ${ }^{2}$ \\ ${ }^{1}$ Institute for Risk Assessment Sciences, University of Utrecht, Utrecht, The \\ Netherlands \\ ${ }^{2}$ Department of Health Care and Epidemiology, University of British Columbia, \\ Vancouver, British Columbia, Canada
}

INTRODUCTION 245

EXPOSURES RELEVANT TO HEALTH: THE CONCEPTUAL

FRAMEWORK 246

Considerations of Exposure Timing 246

Considerations of Exposure Route 247

Practical Context of Pesticide Exposure 248

EPIDEMIOLOGICAL STUDY DESIGNS AND EXPOSURE

ASSESSMENT 248

Prospective Cohort Studies 248

Retrospective Cohort Studies 249

Case-Control Studies 250

Cross-Sectional Studies 252

EXPOSURE ASSESSMENT STRATEGIES 252

INFLUENCE OF THE ACCURACY OF EXPOSURE PROXIES ON

MEASURES OF ASSOCIATION 254

Errors in Qualitative Proxies 254

Errors in Quantitative Assessments Versus Variability 255

EXPERIENCE WITH EXPOSURE ASSESSMENT

IN CASE-CONTROL STUDIES 259

Occupational Histories 260

Exposure Matrices: Using Job or Crop to Infer Exposure 261

Self-Reported Exposures 261

Expert-Reviewed Exposures 262

Determinants of Exposure Studies 264

Exposure Databases 264

Exposure Measurements 265

CONCLUSIONS AND RECOMMENDATIONS 266

REFERENCES 268

\section{INTRODUCTION}

Epidemiology is the science that involves the study of the distribution and determinants of disease. With regard to pesticide exposure, to workers through

Occupational and Residential Exposure Assessment for Pesticides. Edited by C. A. Franklin and J. P. Worgan (c) 2005 John Wiley \& Sons, Ltd ISBN: 0-471-48989-1 
occupational use or to people in their homes through direct or indirect exposure, the research considers the role of pesticides in the etiology of a number of diseases, ranging from acute poisoning and neurological effects to cancers and reproductive outcomes. The results of epidemiological studies can be used for development of preventive strategies as part of public or occupational health programs, especially when quantitative exposure information has been used and exposure-response relationships have been evaluated.

This chapter will focus mainly on the derivation of occupational exposure values and the special challenges it poses to epidemiologists studying the health effects of workers exposed to pesticides. Despite the fact that occupational exposures are our focus, the issues introduced in this chapter are also relevant to studies of environmental health risks from pesticides, to farmers' family members, although studies among this category of exposed individuals are still relatively exceptional. Exposure of family members during low-exposure tasks is likely to occur (De Cock et al., 1998a,b; Arbuckle et al., 2004a; Hogenkamp et al., 2004). In addition, domestic exposure to detectable residues of pesticides in homes of farmers is an area of concern (Loewenhertz et al., 1997).

First, this chapter will describe a conceptual framework to illustrate the special challenges posed because exposures assessed for epidemiologic studies must be relevant to the health outcome under investigation. Secondly, some of the most commonly applied epidemiological study designs will be introduced, with special emphasis on exposure assessment issues associated with the design. Thirdly, some widely applied exposure assessment approaches will be introduced, ranging from qualitative classifications of exposure to quantitative exposure assessment of pesticide concentrations. The influence of measurement error on measures of association between exposure and disease, such as the slopes of exposure-response relationships and risk or odds ratios, will be briefly reviewed. Finally, exposure proxies used in case-control studies of chronic effects of pesticide exposure will be reviewed and the concepts introduced earlier will be applied.

\section{EXPOSURES RELEVANT TO HEALTH: THE CONCEPTUAL FRAMEWORK}

Considering pesticide exposure in the context of epidemiological investigations involves the evaluation of exposures that are relevant to health. Exposure is usually defined as contact with an agent and can be contrasted with absorbed dose, the amount that enters or interacts with the organism. The concept of health-relevant exposure implies that not all exposures lead to, or are associated with, a certain health risk.

\section{CONSIDERATIONS OF EXPOSURE TIMING}

An important dimension of health-relevant exposure is the time axis. For instance, when studying cancer risks, current exposures to pesticides are usually not relevant, but exposures in the past may be important. Although exposure might 
be ongoing, the more recent exposures might be omitted in an epidemiological study of carcinogenic effects of pesticide exposure. The terminology for this phenomenon is the health-relevant 'time-window' of exposure. The time-window differs for different health endpoints. For instance, evaluation of the effects of pesticide exposure on the semen quality of sprayers involves a considerably shorter time-window, perhaps as short as one cycle in spermatogenesis.

The combination of the health-relevant time-window and the toxicokinetic properties of the agent of interest determine the optimal exposure assessment strategy. Dioxin, a contaminant of chlorophenoxy herbicides and fungicides, has a relatively long biological half-life, estimated at about seven years and is measurable in serum. Serum measurements of dioxin are therefore relatively stable, and simple first-order kinetics have been used to 'back-estimate' serum dioxin levels on the basis of an occupational history. Such exposure data have been used quite successfully in epidemiological analyses of cohorts of pesticide producers (Hooiveld et al., 1998).

Nowadays, in Western countries in particular, pesticides with shorter biological half-lives are being used. Captan is an example of a well-known fungicide with a half-life in the environment of 10 to 17 days. The half-life in the body is shorter; most captan is metabolized and excreted within $48 \mathrm{~h}$. Therefore, measurements in urine are only representative of exposure for a short period after exposures during application or re-entry activities. In addition, exposure during re-entry only occurs in the first few weeks after fungicide application since captan is also not persistent in the environment. An epidemiological study in which the exposure assessment is based on evaluating the level of tetrahydrophthalimide (THPI), an easily measurable metabolite of captan, will involve complex logistics and will in most cases be impractical, since timing of the measurements need to be closely related to the activities of the farmers. Moreover, a single measurement of THPI is likely to be a poor predictor of the long-term average captan exposure because of the short half-life, and thus of little use when chronic effects are of interest.

\section{CONSIDERATIONS OF EXPOSURE ROUTE}

The route of exposure is another aspect of exposure in which health-relevance must be considered. In Section One of this book, there is a detailed discussion of exposure assessment methodologies, including the importance of identification of the most prevalent route of exposure (dermal, inhalation or oral) and the necessity of knowing the absorption of the pesticide to allow calculation of the absorbed dose for risk assessment. For epidemiological purposes, exposureassessment studies are usually limited to assessing contact exposure levels. Since dermal absorption is not known for many pesticides or complex mixtures, uptake through the dermal route can often not be estimated and contact exposure data are a poor proxy of internal exposure (absorbed dose) (Schneider et al., 1999).

These factors illustrate the complexity of exposure assessment for epidemiology. The need to consider both the health-relevant time window and the biological 
aspects of exposure and dose may lead to complex logistics and creative methods to estimate internal dose. In diseases with long induction and latency periods, the opportunity to quantitatively measure relevant exposures may simply have passed before epidemiological studies are initiated.

\section{PRACTICAL CONTEXT OF PESTICIDE EXPOSURE}

Most agricultural workers use numerous pesticides over a growing season. Depending on the crop, some pesticides are applied in combinations (tank mixes) or are applied at different times during the season. As a result, epidemiological studies on the health effects of occupational pesticide exposure evaluate the effects of these mixtures. Attribution of health effects to any single pesticide is difficult, if not impossible. Few studies among pesticide workers establish the effect of exposure to one or a mixture consisting of a limited number of pesticides.

\section{EPIDEMIOLOGICAL STUDY DESIGNS AND EXPOSURE ASSESSMENT}

It is conceptually easiest to introduce all epidemiological study designs by starting with the cohort study design.

\section{PROSPECTIVE COHORT STUDIES}

In a prospective cohort study, disease-free exposed and unexposed individuals are selected and followed concurrently over time to determine whether or not they develop the disease(s) of interest. This type of study requires enumeration and follow-up of the workers involved. The risk of developing the disease (i.e. disease incidence) is usually calculated as the number of individuals who develop the disease during follow-up, divided either by the number of individuals at baseline (the source population), or the accumulated number of 'person-years' at follow-up (Figure 7.1). The latter measure is preferred since it is less sensitive to differential loss to follow-up in diseased and disease-free subjects, but also compensates for a potentially different disease incidence in the exposed and unexposed due to other external factors than the exposure under study. The background incidence of the disease of interest is based on observations among unexposed controls. The difference in incidence between exposed and unexposed is usually expressed as a ratio, the 'risk ratio' or 'relative risk' (RR), although other expressions can be used. Since a sufficient number of disease cases will only be accumulated after a reasonable follow-up time, prospective cohort studies are relatively expensive and time-consuming. The advantage, on the other hand, is that all exposure assessment options are available; investigators can decide to assess the exposure to any agent of potential relevance in a quantitative way, and repeated over time if desired. 


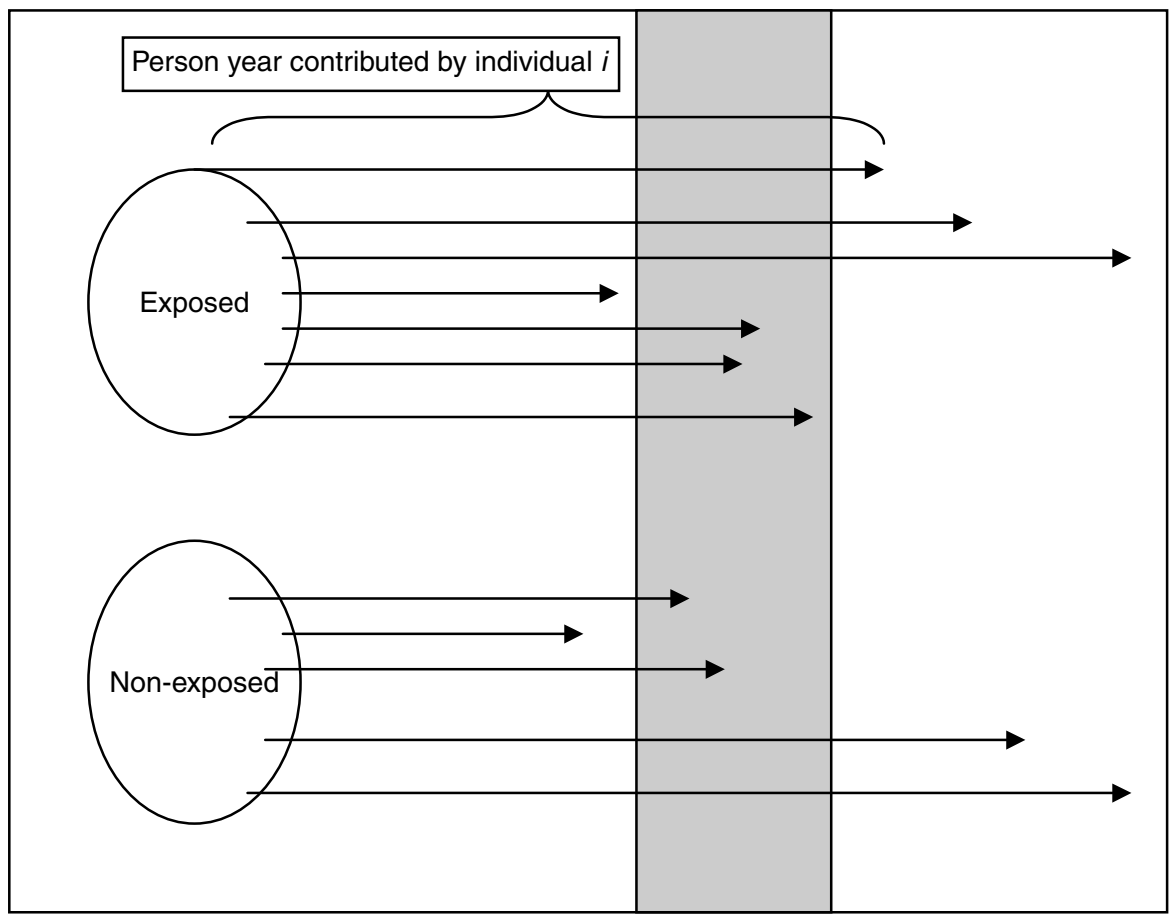

Time $\longrightarrow t_{0}$

$t_{1} \quad t_{2}$

Figure 7.1 The design of a cohort study and its relationship with case-control study designs. Individuals who died during follow-up in the shaded area are eligible for inclusion in a case-control study including incident cases between $t_{1}$ and $t_{2}$. The controls in the case-control study are individuals who did not have the disease of interest at the time of involvement. Arrows indicate end of follow-up, either because of (a) loss to follow-up, (b) mortality due to cause of interest, or (c) other causes of death. The risk ratio, or relative risk $(\mathrm{RR})=(\#$ died cause of interest in exposed $/ \Sigma$ person years $) /(\#$ died cause of interest controls $/ \Sigma$ person years)

\section{RETROSPECTIVE COHORT STUDIES}

A more efficient form of the cohort study is the retrospective cohort design. The study population must be identified through personnel files or other available historical information. Depending on the information available, individuals exposed and even deceased decades ago may be enrolled in the study. A long follow-up period allows evaluation of excess risks even for diseases with a relatively low incidence. Morbidity or mortality outcomes must be established through disease registries or national mortality statistics, as they are for prospective cohort designs. A retrospective study, including identification of the cohort and completion of the disease follow-up can be completed in one or a few 
years, depending on the local data sources. The disadvantage is that data about past exposures are usually not available, and so exposure estimates have to be based on often poorly described measurement series, expert opinions, worker reports, or sometimes even reconstructions of exposure processes. In many retrospective cohort studies, only qualitative information related to exposure is available through knowledge of the job titles or departments in which the person worked. In rare instances, the agent under study may be persistent in the body and can be measured in serum or another body fluid, as in the dioxin example described above.

\section{CASE-CONTROL STUDIES}

The case-control design is different from the cohort design, but clearly related. Cases arising during follow-up can be randomly matched to controls from the same cohort, but without the disease of interest at the time the case occurs. Figure 7.2 shows the basic elements of a case-control design. The goal of the investigation is then to identify whether the pesticide exposure histories of cases differ from those of the controls. The most important aspect of this approach is that no information about the source population is needed to derive an estimate of the relative risk, called the 'odds ratio'. The relative risk is estimated by the ratio of the odds of exposure in cases divided by the odds of exposure in controls. Mantel-Haenszel or logistic regression methods can be used to calculate odds ratios while adjusting for potential confounding factors. The design described here, a case-control study within a cohort, is usually referred to as a nested casecontrol study. This approach is used because the design is more efficient than a cohort study. More effort can be used to evaluate historical exposures in a very detailed way in a small number of study subjects, and the estimated risk ratios will be similar.

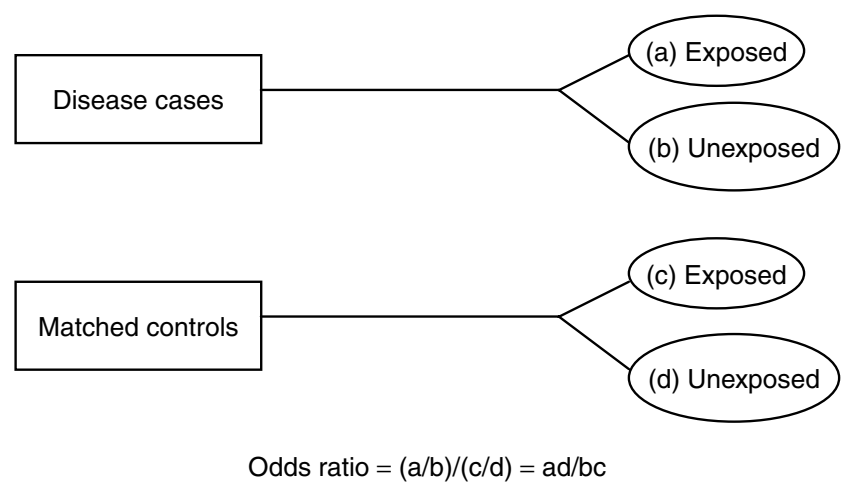

Figure 7.2 Schematic of the design and analysis of a case-control study 
The most interesting aspect of the case-control design study is that participants do not necessarily need to be enrolled through a cohort study. Participants can also be identified through disease registries or medical records. Controls representing the population from which the cases arose are selected at random, with matching on criteria such as age and sex, which may affect disease distribution independently of the exposure of interest. This type of case-control study, referred to as 'population-based', is especially efficient when relatively rare events are being studied.

An advantage of case-control studies is their relatively low cost when compared to cohort studies, especially when investigating rare diseases, since only a sample of the population of interest is enrolled as controls, rather than the entire cohort, which generated the cases. For exposures such as pesticides, which may occur in widely dispersed segments of the population, including manufacturers, farmers, crop harvesters, food packers and processors, pesticide applicators, florists, landscaping personnel, silviculture workers and others, a populationbased case-control design theoretically allows examination of a broad range of exposure levels and should be logistically simpler than assembling a cohort when the exposed individuals are scattered in small work groups. Case-control studies will have the greatest power to examine the effects of pesticides when they are based in locations such as agricultural communities where the proportion of the population exposed is high. They also offer the opportunity to enumerate multiple exposures, including both occupational and residential exposures, as well as medical and lifestyle factors that may be confounders of the pesticide-disease association.

Despite the advantages of case-control studies, exposure assessment remains the most problematic element. Recent reviews of pesticide studies indicate that exposure data are usually gathered by interviewer-administered questionnaires, or occasionally from mailed questionnaires or medical records (Maroni and Fait, 1993; Bohnen and Kurland, 1995; Daniels et al., 1997). Exposures enumerated from these sources are not quantitative measurements, but subject- or proxy-reported job histories, tasks, residence locations, or recalled exposures to pesticides (Bohnen and Kurland, 1995; Daniels et al., 1997; Zahm and Ward, 1998). More sophisticated techniques for assessing exposures in case-control studies are available, but to date only rarely employed. The validity and reliability of recall is therefore an essential question in considering the results. In a study in which the level of the herbicide 2,4-D was known, the sensitivity of self-reported use was only $56.7 \%$ with a specificity of $86.4 \%$; it was much higher (sensitivity $91.6 \%$ and specificity 67.4 , respectively) for another herbicide, MCPA (Arbuckle et al., 2002). However, the relevant question to ask is not only what the accuracy is of a certain exposure characterization methodology, but also what the effect of imperfect accuracy is on measures of association in epidemiological studies. This has been an area of major development over the last decades and is described in the following sections. 


\section{CROSS-SECTIONAL STUDIES}

Another common design used in pesticide epidemiology is the cross-sectional study. As implied by the name, these studies evaluate exposures and disease status simultaneously at one point in time, usually at the work site of interest. This design is used for investigating diseases which are not routinely reported, and therefore require measurements to allow cases to be identified. An example is respiratory disease that involves lung function measurements. Relative risks can be estimated by comparing the disease prevalence in subjects with differing levels of exposure (note: prevalence is the number of existing disease cases, and is not the same as disease incidence). A major flaw of this study type is its inability to identify the temporal relationship between the exposure and the disease, i.e. did the exposure take place prior to disease development or not? Related to this issue is the concern that individuals who are most susceptible to an exposure may leave the workforce early, and therefore may not be present to be enrolled in the study. An advantage of cross-sectional studies is that quantitative exposure measurements can be made at the work site during the study period. However, investigators must be conscious of whether these measurements adequately estimate exposures in the health-relevant time-window.

Additional details on the design and analysis of epidemiological studies can be found in standards texts such as Checkoway et al. (2004) and Rothman and Greenland (1998).

\section{EXPOSURE ASSESSMENT STRATEGIES}

As indicated by some of the examples described earlier, epidemiological studies can use a wide range of approaches for characterizing exposure to chemical agents such as pesticides (Table 7.1).

Qualitative information on exposure can be obtained from study subjects using questionnaires or interviews. Experts such as occupational hygienists can evaluate the exposure by 'walk-through' surveys, detailed workplace investigations using validated checklists or judgements based on their experience.

Quantitative exposure assessment is possible by measuring the external exposure in air or on skin, or by measurements of biological markers in serum, urine, fat or other relevant body fluids.

Quantitative exposure data can be used in different ways, as follows:

- to confirm that exposure has occurred (absorbed);

- to directly estimate the exposure of study subjects;

- to validate qualitative or semiquantitative exposure estimates;

- to predict exposures on the basis of empirical modeling.

Direct exposure assessment using quantitative exposure estimates can involve different strategies, ranging from assessing the exposure for each participant by using repeated measurements over time to grouping strategies that categorize 


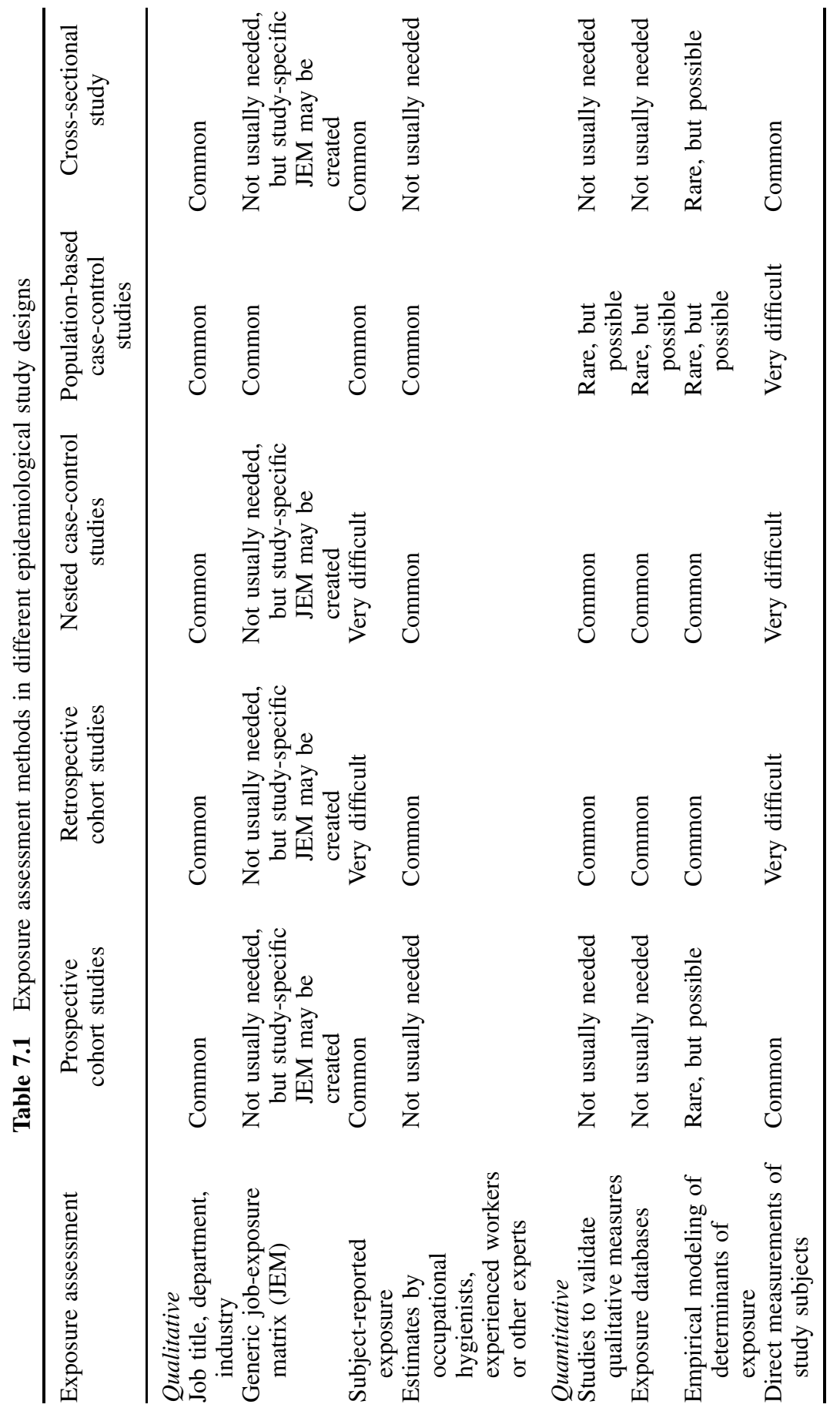


workers into homogeneous exposure categories (Rappaport, 1991). Some examples of validation studies exist (de Cock et al., 1998a,b; London and Myers, 1998) but there is a need for more. Empirical modeling studies show that information on determinants of exposure, such as contamination of foliage or time since the last spraying of an orchard, can be quantitatively associated with dermal exposure and might be used to group workers into high- and low-exposure categories (Brouwer et al., 1994; Tielemans et al., 1999b). However, few examples exist of application of these principles in epidemiological studies. De Cock et al. (1994) grouped fruit growers in different exposure categories based on information regarding type of spray, use of a vehicle cab during spraying, size of the orchard, etc., in a study on time to pregnancy and pesticide exposure. Their grouping was validated by detailed exposure measurements of the pesticide in the air or on the skin of the worker.

Before examining each of these exposure assessment methods in more detail, it is important to consider the consequences of imprecise or biased exposure assessment on the risk estimates made in an epidemiological study.

\section{INFLUENCE OF THE ACCURACY OF EXPOSURE PROXIES ON MEASURES OF ASSOCIATION}

The issue of measurement errors has received considerable attention in the field of epidemiology. This is because measurement errors influence the estimates of association between exposure and disease and can obscure or inflate the true exposure-response relationship. Therefore, the accuracy of exposure assessment should be an integral element of any etiological epidemiological study and needs to be reviewed before final conclusions can be drawn.

The accuracy of exposure assessment is determined by systematic and random errors in the assessment. For quantitative exposure assessments, important sources of error include measurement errors (i.e. from laboratory and field monitoring techniques), as well as variations in exposure over time and space. For qualitative exposure proxies (e.g. self-reported past exposures, occupational histories or expert evaluations), the most important sources of error are recall bias (systematic differences in exposure recall between cases and controls) and random error, expressed in terms of intra- and inter-rater agreement. Although systematic errors can result in serious misinterpretations of the data, especially due to scaling problems, random errors have received more attention in epidemiology because this type of error is pervasive, and its effect is usually to diminish estimates of association between exposure and disease. The magnitude of random errors can be considerable in epidemiological field studies.

\section{ERRORS IN QUALITATIVE PROXIES}

Although it is difficult to generalize, the validity of qualitative proxies of exposure can be very poor in some cases. Tielemans et al. (1999a) recently compared 
qualitative exposure proxies obtained by self-reports, expert judgements, simple checklists and job-exposure matrices to measurements of solvent and metal exposures in a general population study. The sensitivity to identify truly exposed individuals by using the qualitative approaches ranged from only 21 to $85 \%$, while the specificity to identify truly unexposed individuals ranged from 34 to $94 \%$. These results corroborate with observations made specifically for the pesticides cited earlier, by Arbuckle et al. (2002). Errors of this magnitude, if random, can lead to considerable error in the estimation of the true exposure-response relationships (Stewart, 1999). In a study on herbicide exposure to farm children, the sensitivity and specificity of a simple questionnaire item on use were 47 and $72 \%$, respectively, for 2,4-D, and 91 and $30 \%$ for MCPA, by using urinary biomonitoring data as the 'gold standard' (Arbuckle et al., 2004a). If the child was outside during any of the herbicide handling activities, the figures for sensitivity dropped, but specificity improved.

Other factors influence the magnitude of the effect of exposure misclassification on estimates of association between exposures and disease. The effect depends not only on the extent of exposure misclassification, but also on the prevalence of exposure in the population studied. Since pesticide exposure prevalence may differ in different populations and is certainly different in general population studies when compared to studies in farming communities, the performance of exposure assessment techniques will vary according to the study context. The specificity determines the bias in risk-ratio situations with a low exposure prevalence. Thus, a poor sensitivity, for instance, the one reported by Arbuckle et al. (2002) for 2,4-dichlorophenoxyacetic acid (2,4-D), may not be problematic in a general population or case-control study, as long as the specificity is sufficiently high.

Exposure misclassification may not be random, but may differ for individuals with and without the disease of interest (differential exposure misclassification). This further complicates the impact of exposure misclassification and may make it impossible to predict the effects without more detailed information about the structure of the errors.

Other special cases of relevance for pesticide exposure exist. Some studies suggest that residential pesticide exposure of farmers and family members may be as high as exposures experienced during re-entry activities. When such a relevant background exposure is present and correlated to the measured exposure, and not dealt with in an epidemiological study, as usually happens, this may result in nondifferential misclassification of the occupational exposure under study. This can lead to over- or underestimation of the association between exposure and disease, depending on the magnitude of the error and the detailed error structure (Loomis and Savitz, 1994).

\section{ERRORS IN QUANTITATIVE ASSESSMENTS VERSUS VARIABILITY}

With regard to quantitative exposure assessment, many investigators focus their efforts on ensuring that errors due to laboratory and field sampling methods 
are minimized. Typical precision of measurement methods range from \pm 5 to $\pm 35 \%$. In contrast, natural variation of the exposure over time and space may be considerably larger. Pesticide applications (i.e. agricultural) that take place outdoors result in larger variability in exposure than those done indoors (i.e. residential, greenhouses, etc.). Day-to-day differences in exposure levels, due to meteorological conditions, ventilation, production volume and other potential determinants, may be as large as 10- to 1000-fold. As an example, in a large study of fruit growers, exposure to captan during application (mixing, loading and spraying) varied by as much as a factor of 750 for respiratory exposures, 300 for dermal exposure of the arms, and 540 for dermal exposure of the wrists (de Cock et al., 1994, 1998b). These wide variations were made up of day-to-day variability in each worker's exposure, as well as variability between workers. Exposure variation during re-entry to the orchards was smaller and could be attributed mainly to day-to-day variability. Interestingly, between-worker differences tended to be larger for dermal exposure than for inhalation exposure, hence suggesting that work style and possibly personal hygiene have a greater effect on dermal exposures. There is a more detailed discussion of variability, and the steps taken into consideration when the data are being used in risk assessment, in Chapter 1.

Data from a large exposure study in fruit growers provide a clear illustration that day-to-day variability is usually larger than the measurement error, including both sampling and analytical errors. In Table 7.2, a breakdown is given of the total variability in exposure (log (dermal captan concentration)) into variance components for different dermal patch areas. Interestingly, the interlocation variability in dermal exposure seems only relevant when the head, sternal area, wrists and arm are taken together. The variance component of 4.5 suggests systematic differences between these patches. A variance component of 1 for interlocation

Table 7.2 Interworker, intertask and intra- and interlocation variability in dermal exposure to captan assessed through hand-rinsing and using dermal patches, in a large population of fruit growers (from De Cock et al., 1998a,b)

\begin{tabular}{|c|c|c|c|c|c|c|c|}
\hline Area & Method & $N^{a}$ & $k^{b}$ & Interworker $^{c}$ & Intertask $^{c}$ & Interlocation $^{c}$ & Intralocation $^{c}$ \\
\hline $\begin{array}{l}\text { Head, sternal } \\
\text { area, wrists } \\
\text { (average), arm }\end{array}$ & Patches & 677 & 126 & 1.0 & 1.4 & 4.5 & 2.8 \\
\hline $\begin{array}{l}\text { Forehead, sternal } \\
\text { area }\end{array}$ & Patches & 339 & 126 & 2.0 & 1.0 & 1.0 & 2.8 \\
\hline $\mathrm{Arm}^{d}$ & Patches & 292 & 126 & 1.0 & 3.5 & 1.0 & 2.7 \\
\hline $\begin{array}{l}\text { Hands (left, } \\
\text { right) }\end{array}$ & Hand-rinse & 348 & 126 & 2.5 & 2.6 & 1.0 & 3.1 \\
\hline $\begin{array}{l}\text { Wrists (left, } \\
\text { right) }\end{array}$ & Patches & 167 & 126 & 1.6 & 3.6 & 1.0 & 3.0 \\
\hline
\end{tabular}


in the rest of the table suggests that no differences in exposure exist between the forehead, sternal area, arms, hands and wrists, respectively. This also suggest that differences in distribution of the exposure over similar body parts (left versus right hands, wrists, etc.) or the same body part (two patches on the same arm) do not seem to be present, or at least do not contribute in a relevant way with this number of samples taken in a large population study. Since these variance components have been obtained by taking repeated samples for each individual, the intralocation components can be interpreted as day-to-day variability and sampling and analytical error in exposure on the patch. This variance component is relatively large.

Such tremendous variabilities illustrate that a single measurement, or even a limited number of repeated measurements, are most likely poor predictors of long-term average exposure. If few measurements have been taken, some individuals' true average exposure will be underestimated, while for others it will be overestimated. This type of error will usually lead to a loss of study power and underestimation of the relationship between exposure and disease. Formulae exist to allow epidemiologists to reduce this bias in the exposure-response relationship to an acceptable level by calculating the number of repeated measurements required per individual, given a certain ratio of the intra- and interindividual variability in exposure. Interestingly, the underestimation is not determined by the absolute magnitude of the day-to-day exposure variability, but by the ratio $(\lambda)$ of the day-to-day variability to the variability in exposure between individuals. This ratio can be regarded as a noise-to-signal ratio, as follows:

$$
b=\beta(1+\lambda / k)^{-1}
$$

where $b$ is the observed value of the empirical regression coefficient for the relationship between a health outcome $(Y)$ given an exposure $(X)$, when $X$ is measured with error, $\beta$ is the true value of the regression coefficient of $Y$ on $X$, $\lambda$ is equal to ${ }_{\mathrm{w}} s_{i}{ }^{2} / \mathrm{b} s_{i}{ }^{2}$ (in which ${ }_{\mathrm{w}} s_{i}{ }^{2}$ is the estimate of intraindividual (day-today) variance in exposure and $\mathrm{b}_{\mathrm{b}} \mathrm{s}^{2}$ is the estimate of interindividual variance in exposure), and $k$ is the number of repeated measurements per individual.

As an example, consider the data on day-to-day and interindividual variability of fruit growers' respiratory and dermal exposure to captan shown in Table 7.3 (de Cock et al., 1998a). The ratio of the 97.5th percentile to the 2.5 th percentile of the exposure distribution $\left(R_{95}\right)$ is usually larger for the intraindividual or dayto-day variability, when compared to the interindividual variability. The variance ratio, $\lambda$, can be calculated from the $R_{95}$ values, since the standard deviation of each exposure distribution is equal to $\ln \mathrm{R}_{95} / 3.92$, and the square of the standard deviation gives the variance. For the respiratory exposure, this results in a variance ratio $\lambda$ of 32.8 , whereas for dermal exposure of the wrist the variance ratio is considerably lower, approximately 3.0. What are the implications of these variance ratios for the number of measurements per study subject? For a bias of less than $10 \%$ (or $b / \beta \geq 0.90$ ), the number of repeated measurements per subject 
Table 7.3 Intra- and interindividual variability in exposure to captan, expressed as $R_{95}$ (ratio of the 97.5 th percentile to the 2.5 th percentile of the exposure distribution) in a large population of fruit growers (from De Cock et al., 1996)

\begin{tabular}{lllcc}
\hline Captan in/on & $N^{a}$ & $k^{b}$ & $R_{5}$, interindividual & $R_{95}$, intraindividual \\
\hline Inhalable dust & 154 & 108 & 3.1 & 541 \\
Wrist & 188 & 133 & 17.3 & 143 \\
Arm & 176 & 127 & 14.2 & 80.8 \\
Sternal area & 184 & 131 & 7.1 & 12.9 \\
\hline
\end{tabular}

${ }^{a}$ Number of observations.

${ }^{b}$ Number of farm workers.

required to obtain a good estimate of the average respiratory exposure is 295 , and of the dermal exposure 27. Needless to say, this is an unrealistic measurement effort that cannot be realized in most practical contexts (Heederik and Attfield, 2000). However, it is not surprising that this measurement effort is required. The day-to-day variability is especially large in these agricultural workers, compared to many other occupational groups, and could be attributed to variable meteorological conditions or differences in work activities such as the number of tank fills, etc. On the other hand, differences between the fruit growers are relatively small, likely because they all perform more or less the same tasks by using a limited range of different technologies. The study design could be optimized by including other workers with captan exposures, but with considerably higher or lower exposure levels. This would increase the interindividual variance, so that the number of repeated measurements per subject could be reduced considerably. Details regarding such design considerations can be found in the literature (Boleij et al., 1995; Heederik and Attfield, 2000).

Variance ratios obtained from studies of many occupational groups and different chemical exposures suggest that individual exposure assessment is rarely efficient (Kromhout et al., 1993). Because of this, another strategy is often used; workers are grouped into homogeneous exposure categories and exposure data are gathered to estimate the group average exposure. This average exposure level is then applied to all members of that group. Remarkably, in most cases, grouping workers will lead to reasonably unbiased estimates of the exposure-response relationship, because the systematic overestimation or underestimation of exposure of some of the group members leads to an error of the 'Berkson' type (Tielemans et al., 1998).

Theoretically, a grouping strategy is optimal, if each exposure category is as homogeneous as possible with regard to the exposure, if as much contrast as possible exists between categories, and if sufficient measurements per category have been taken so that the estimate of the average exposure in each category is sufficiently precise. This will be accomplished by minimizing the interindividual variability within an exposure category (intragroup variability), maximizing the contrast between groups (intergroup variability) and minimizing the standard error 
of the mean in each exposure category. These requirements may be conflicting because there are always only a limited number of measurements available. Making categories as homogeneous as possible will generally lead to a large number of small categories of workers with similar occupations or tasks. In the extreme case, the grouping strategy becomes an individual exposure assessment strategy when each individual is in a separate category. This, in turn, reduces the number of measurements per exposure category and thus the precision of the estimate of the average exposure. This apparent conflict between several requirements can only be solved by using quantitative expressions for calculating optimum grouping strategies, as already exist for individual-based exposure assessment strategies. Some formulae have recently been published for balanced data sets assuming a constant variance over and within exposure categories (Tielemans et al., 1998). Expressions applicable to a broader range of conditions are not yet available.

Some of the above-described problems with quantitative exposure assessment, combined with the logistical difficulties that are introduced when one wants to apply quantitative assessments to populations of agricultural workers (especially difficult for distant past exposures), probably explain why so few epidemiological studies use measurement data. In addition, differences between workers in the agricultural sector might be somewhat less when compared to the industry because often similar tasks are performed with relatively comparable technology. This implies that one is interested in exposure contrasts with a relatively homogeneous exposed population. It is unfortunate that so few quantitative exposure data are available, because as a result, epidemiological studies can often not be used in risk assessment or to set exposure standards. When only simple qualitative proxies of exposure, such as job title or agricultural activity, have been used, inferences about the levels of exposure responsible for adverse health effects are difficult or even impossible.

\section{EXPERIENCE WITH EXPOSURE ASSESSMENT IN CASE-CONTROL STUDIES}

Maroni and Fait (1993) reviewed the literature on the chronic health effects of pesticides over the period from 1975 to 1991, and found that case-control studies were the most common design, representing about $47 \%$ of epidemiological studies. A search of the 'Medline' bibliographic database from 1992 to 1999 showed that the proportion of case-control studies remains equally high. The large majority of these studies use a population-based, rather than nested, case-control design. This is likely because the health effects that have dominated pesticide research, in particular adult and childhood cancers and reproductive outcomes, including birth defects (Maroni and Fait, 1993), are rare events that are more easily examined by using this study type.

As mentioned earlier, population-based case-control studies have many advantages; however, exposure assessment is generally considered a fundamental 
weakness of these designs. Quantitative exposure assessment methods are rarely used, and only in recent years have the reliability and validity of typical qualitative assessment methods been examined. Because of the unusual nature of the 'exposure assessment' methods used in case-control studies, it is worthwhile to consider their strengths and limitations here. The following sections will consider in more detail these methods: occupational histories, exposure matrices, self-reported exposures, expert-reviewed exposures, determinants of exposure studies, exposure databases and direct exposure measurement. As indicated in Table 7.1, most of these methods may also be used in other types of epidemiological studies.

\section{OCCUPATIONAL HISTORIES}

In the initial exploratory work examining potential associations between an exposure and disease, analyses comparing the relative risks across occupations or industries may point to exposures requiring further study. Data on job and industry in case-control studies, whether from medical records or questionnaires, are usually derived from self-reports or, when a subject is dead or in some way incapable, reports by next-of-kin. A great advantage of using occupation and/or industry as the 'exposure' is that there is consistent evidence in comparisons with company, pension and union records with earlier self-reports that occupational histories are well recalled by study subjects (levels of agreement from 70 to $90 \%$ ), although there is a tendency to recall more recent and usual jobs more accurately than past or shortterm jobs (Baumgarten et al., 1983; Rosenberg et al., 1987; Stewart et al., 1987; Bond et al., 1988; Bourbonnais et al., 1988; Rona and Mosbech, 1989; Brisson et al., 1991; Wärneryd et al., 1991; Brower and Attfield, 1998)

In open-ended questioning, next-of-kin report occupational histories somewhat less well, reporting fewer jobs, especially omitting those early in a subject's working life (Pershagen and Axelson, 1982; Pickle et al., 1983; Lerchen and Samet, 1986; Rocca et al., 1986; Boyle et al., 1992; Schnitzer et al., 1995). If there are more next-of-kin interviews (proxies) for cases than controls, it is possible that risk estimates will be underestimated for jobs early in cases' lives. Several studies have specifically examined proxy reporting of agricultural jobs (Johnson et al., 1993; Wang et al., 1994) and found 85 to $93 \%$ agreement between next-of-kin and subjects on prompted questions about 'ever farmed', 'agricultural work' and 'field crop farming'. The level of agreement did not differ between cases and controls, hence suggesting little likelihood of recall bias arising from prompted reports of occupation by next-of-kin.

The difficulty with analyses by occupation and industry is that they do not identify specific agents as risk factors. For example, farmers may be exposed to pesticides, but they also have potential for exposures to other agents, including fuels, solvents, welding fumes, wood dust, silica, crop dust, animal danders, zoonoses and endotoxins. In addition, although some farmers use pesticides, many others do not (Blair and Zahm, 1993). An elevated risk in a job can only be suggestive of particular exposure risks. 


\section{EXPOSURE MATRICES: USING JOB OR CROP TO INFER EXPOSURE}

In an effort to utilize the accurate recall of occupational information, but overcome its indirect connection to exposures, there was a movement in occupational epidemiology in the 1980s to develop job-exposure matrices (JEMs). The latter list a wide range of jobs on one axis and a wide range of exposures on the other, while the cells of the matrix indicate the intensity, frequency and/or probability of a specific exposure in a specific job. A number of generic JEMs, made publicly available, were created by using either expert judgement, often aided by published literature (Hoar et al., 1980; Pannett et al., 1985), or 'walkthrough' surveys of a representative sample of work sites (Sieber et al., 1991). Studies comparing exposure assessments between these JEMs have found poor agreement (Linet et al., 1987; Kromhout et al., 1992; Hawkes and Wilkins, 1997) as have studies examining the comparability of JEMs and self-reports (Kromhout et al., 1992; Roeleveld et al., 1993; Rybicki et al., 1997) or JEMs and study-specific expert assessments (Bouyer et al., 1995; McNamee, 1996; Rybicki et al., 1997). The problem seems to stem from the often very poor sensitivity of generic JEMs, understandable given the number of cells which need to be evaluated, and the sometimes unpredictable circumstances in which chemicals may be used.

Most JEMs do not include pesticide exposures or agricultural work in the matrix. Recently, a number of investigators have developed exposure matrices directly applicable to agricultural work (Duares et al., 1993; Miligi et al., 1993; Nanni et al., 1993; London and Meyers, 1998). A unique feature of these matrices it that experts from the agricultural industry and occupational hygienists have used not only job, but also crop and other information as the basis for assigning exposures. In addition, these matrices are more study-specific, and have attempted to assign a limited number of agricultural chemical exposures to a limited set of jobs or crops, therefore increasing the likelihood that the experts are knowledgeable about the factors they are rating. The crop-exposure matrix of Miligi et al. (1993) had sensitivities ranging from 0.83 to 1.0 and specificities ranging from 0.66 to 0.96 when compared to self-reports - a substantial improvement over the results from generic JEMs. However, organophosphate exposure estimates derived by the London and Meyers (1998) matrix explained only $5 \%$ of the variability in erythrocyte cholinesterase levels in a small sample of their study population. More validity and reliability data are required to evaluate the utility of such matrices.

\section{SELF-REPORTED EXPOSURES}

Questionnaires used in case-control studies now commonly ask about more than a subject's occupational history, querying use of pesticides as a group, classes of compounds or specific-tradename products or active ingredients. Investigations of environmental risks from pesticides most often use questionnaire self-reports of pesticide use in gardens, on pets and in homes, as well as potential exposures from drinking water, contaminated food, agricultural drift or contaminated clothing. 
Studies examining the ability of subjects to accurately report their exposures indicate that the quality of responses is directly linked to the type of questioning. Improvements occur with prompted over open-ended questioning, with chemical names used in the work site over the names of chemical constituents, and with compounds that can be sensed over those which are odorless and invisible (Ahlborg, 1990; Joffe, 1992; Blair and Zahm, 1993; Teschke et al. 1994; Nieuwenhuijsen et al., 1997).

Blair and Zahm (1993) postulated that farmers may be more likely than others to recall the use of specific pesticides and their active ingredients, because their use is such a critical component of the success of farming operations. In their study comparing farmers' self-reported use of 'herbicides' and 'insecticides' to suppliers' records, the level of agreement was about $60 \%$. Agreement about years of pesticide use ranged from 38 to $68 \%$. The suppliers' records were not considered a 'gold standard', and therefore the validity of the self-reported information was expected to be greater than suggested by these results. The levels of agreement were similar for cases and controls, thus suggesting recall bias is unlikely. In a similar study of structural fumigation workers, Calvert et al. (1997) found Pearson correlation coefficients of 0.97 for years of employment in the company, and 0.66 to 0.88 for percentage of jobs using two specific fumigants, but lower for days worked. Population-based studies (Eskenazi and Pearson, 1988; van der Gulden et al., 1993) showed 82\% agreement in repeated self-reports of exposure to the broad category 'pesticides'. In populations less involved with pesticide application, such as migrant farmworkers or those living in residences which have been treated, there is evidence that few know the names of specific pesticides to which they have been exposed (Zahm and Blair, 1993).

Evidence about next-of-kin reports of subjects' exposures is mixed. Some studies of farmers and their wives querying the use of specific pesticides (Blair and Zahm, 1993), as well as population-based samples querying pesticide use in general (Boyle et al., 1992), indicated that next-of-kin under-reported exposures. Other studies have reported rather good concordance between subjects and next-of-kin, with agreements of 70 to $95 \%$ for use of 'pesticides', 'herbicides', 'insecticides' or 'fungicides' (Johnson et al., 1993; Wang et al., 1994). In most studies, pesticides that were less frequently used were less well known by next-ofkin, as were details about frequency of use, or specific pesticides. Studies which examined the next-of-kin's recall separately for cases and controls found once again that there was little difference in recall patterns by case status (Johnson et al., 1993; Wang et al., 1994).

\section{EXPERT-REVIEWED EXPOSURES}

It is difficult for subjects reporting their own exposures to consider their exposures in relation to those of other subjects in the study (Teschke et al., 1989). For example, office workers whose building was sprayed with insecticides might consider themselves exposed, but might not give the same answer if asked to 
compare their exposure to that of a pesticide applicator. As a mechanism for overcoming this problem, it is now a common feature of case-control studies to include a review of self-reported information by occupational hygienists or other experts.

Studies examining agreement between experts' ratings of pesticide exposures have shown good concordance. An overall weighted kappa of 0.76 was measured for two agricultural chemists ranking exposure to phenoxy herbicides, based on subject-reported jobs, farm locations, crops and pesticide handling (Ciccone and Vineis, 1988). A kappa of 0.75 was found for repeated assessments of exposure to 'agricultural chemicals' by four industrial hygienists, based on subject-reported occupational histories (McGuire et al., 1997). Intraclass correlations of 0.88 to 1.0 were reported for eight agronomists rating exposures to 'fungicides' and 'insecticides' in vine growing, based on subject-reported job title and pesticide exposures (Segnan et al., 1996). These excellent results were not sustainable for specific pesticides unless the experts were supplied with product lists from the farms. Somewhat lower levels of agreement were observed in a study examining pairs of hygienists' ratings of sawmill fungicide exposure based on job-title information (intraclass correlations of 0.40 to 0.68 ) (Teschke et al., 1989), and a study of groups of five experts' assessments of captan exposure based on task, equipment and weather information (intraclass correlations of 0.53 to 0.81 ) (de Cock et al., 1996). One study examined the agreement between subject-reported and hygienist-assessed exposure to 'pesticides' (Rodvall et al., 1996). The kappa was 0.88 for cases and 0.46 for controls, in one of the few studies suggesting a basis for concern about recall bias. Controls were more likely to report pesticide exposure when the hygienist assessed none.

The validity of experts' ratings has also been examined. Segnan et al. (1996) found that experts' attribution of 'fungicide' exposure had high sensitivities (0.99) when compared against farm records of pesticide use. The sensitivities for 'insecticides' were lower ( 0.54 to 0.61 ), but the specificities improved when the experts used additional information on pesticide use from study subjects (from 0.60 to 0.99). The validities of specific pesticide ratings were very poor, such that risk estimates could be reversed because sensitivities and specificities added to less than 1.0 (Flegal et al., 1986). In studies using exposure measurements as the 'gold standard', the Pearson correlation coefficients ranged from 0.28 to 0.51 when comparing hygienists' ratings to urinary chlorophenol measurements (Teschke et al., 1989), while the Spearman correlation coefficients for experts' rankings of captan exposure levels ranged from -0.25 to 0.9 (de Cock et al., 1996). These studies highlight the need to consider the accuracy of 'experts'. All of the validity studies considered exposures within narrow occupational settings, unlikely to mimic the range of jobs being assessed in case-control studies. Determining the validity of both self-reported exposures and experts' assessments in the typical population-based setting of case-control studies remains a difficult problem, without study-based exposure measurements, as discussed below. 


\section{DETERMINANTS OF EXPOSURE STUDIES}

A method which holds promise for improving the validity of exposures assessed by questionnaires is to guide the formulation of questions and interpretation of their answers with studies which evaluate the factors affecting pesticide concentrations (Blair and Zahm, 1990). Determinants of exposure studies include experimental and observational studies, which measure exposures and concurrently document work or residence characteristics which may increase or reduce exposure levels (Burstyn and Teschke, 1999). There is a large and growing literature on pesticide exposures, in the mixer, loader and applicator population which is routinely examined for pesticide registration purposes, but also in populations exposed in other environments, including harvesting, residences, silviculture and elsewhere (van Hemmen, 1992; Stewart, 1999). Factors which have been examined as determinants of pesticide exposure are extremely varied, for example, method of application, surface area treated, weather conditions, ventilation, tractor enclosure, tasks, crops, region, protective clothing, time since application, cleaning and laundry facilities, pesticide storage, proximity of residences to mixing and spraying operations, and work practices. One particular study evaluated the effectiveness of personal protective equipment in an observational study (Arbuckle et al., 2004b). However, the exposure-determinant exposure studies conducted so far suggest that the known determinants explain the variability in exposure only to a limited extent. More studies and more developmental work are needed to optimize this approach and make it applicable in an epidemiological context.

Moreover, translating these data into questions useful to assess exposures in case-control studies is not a simple process. Given that data on exposure determinants are likely not to have been collected in the worksites or residences of the study subjects, it would be necessary to consider the transferability of the information. Where studies show consistent patterns and greater variability between the determinants of interest than between sites, useful questions might be developed to distinguish exposure levels. Questions about exposure determinants would have to be answerable by study subjects, suggesting that determinants such as tasks may be much more useful than technical ones such as air-flow rates of greenhouse ventilation systems. Where insufficient information is available in the literature, researchers might consider designing their own determinants of exposure studies prior to embarking on the epidemiological investigation. Tailor-made determinants studies, underpinned by studies testing the validity of subjects' questionnaire responses, would be the ideal method to optimize exposure estimates. Some interesting examples exist of studies which have measured exposures in large numbers of worksites to create predictive models for use in epidemiological studies (Preller et al., 1995; Burstyn and Teschke, 1999).

\section{EXPOSURE DATABASES}

Another method to help quantify pesticide exposures is the use of exposure databases. The exposures measured for pesticide applicator studies are now 
routinely included in the Pesticide Handlers Exposure Database (PHED) database in North America (Leighton and Nielsen, 1995) and POEM and others in Europe (see Chapter 5). They contain data on the dermal and respiratory exposures of mixers, loaders, applicators and flaggers required for pesticide registration, but not of other occupations. In addition, they do not identify the data by the specific chemical name but do indicate the class of pesticide (i.e. herbicide, insecticide, etc.) and the formulation type. A major limitation of several of the abovementioned databases is that the data are collected for pesticide registration and describe exposure under relatively ideal circumstances and a limited number of exposure scenarios. Data from observational studies that measure exposure under 'real-life' conditions are not included, and therefore exposure estimates based on these databases may underestimate both exposure levels and variability.

Other data sets, with a broader coverage of jobs throughout the population, are likely to be needed for estimation of pesticide exposures in case-control studies. For example, Stewart and co-workers (Stewart and Stewart, 1994; Stewart et al., 1998) have used detailed occupational questionnaires with job-specific modules, together with data from the US Occupational Safety and Health Administration (OSHA) Integrated Management Information System to aid in assigning study subjects' exposures to multiple chemicals, including pesticides.

The basis for assignment of exposures is limited by the supplementary information included in the database. The OSHA data set, for example, contains information on job and industry but not tasks or other determinants. Proposals for broadening the data contained in these administrative databases could make them useful for determinants of exposure studies, with the promise and caveats stated above (Rajan et al., 1997).

\section{EXPOSURE MEASUREMENTS}

An avenue for both occupational and environmental exposure assessment which has only rarely been used in case-control studies is direct exposure measurements of the study subjects. For pesticides with long biological half-lives, and whose concentrations are unlikely to be affected by the disease, biological measures of exposure can be made. For example, Caldwell et al. (1981) and Scheele et al. $(1992,1996)$ measured pesticide levels in bone marrow and serum in adult and childhood cancer cases and controls.

For outcomes with short induction and latency periods, measurements of current exposures may serve as reasonable surrogates of exposure in the induction period. Floderus et al. (1993), in a case-control study of brain cancer and leukemia, made 924 measurements of magnetic fields in 169 jobs held longest in the workplaces of study subjects. Similar comparisons have been made for exposure to solvents and heavy metals in a study on reproductive outcomes (Tielemans et al., 1999a; Stewart, 1999). Given advances in occupational hygiene monitoring equipment over the last two decades, it is reasonable to consider mailing simple sampling equipment, such as passive dosimeters or electronic data loggers, 
to study subjects for exposure assessment. If these avenues of exposure assessment are adopted in case-control studies, the issues involved will be similar to those faced by researchers using quantitative measures, as discussed earlier in this chapter.

\section{CONCLUSIONS AND RECOMMENDATIONS}

A wide range of exposure assessment approaches is available and the guiding concepts can be found in the epidemiological and occupational and environmental hygiene literature. However, exposure assessment of pesticides in epidemiological studies is still in its infancy, as shown by a review of the literature on exposure assessment in case-control studies. Most exposure proxies are, at their best, qualitative, and discriminate only between exposed and unexposed subjects. There is a clear need for validation of questionnaire self-reports and expert-based proxies of exposure using quantitative data gathered under 'real-life' conditions and describing the variability in exposure over time and space. Quantitative pesticide exposure studies have seldom been applied in epidemiology - another opportunity for improvement. A complication in this field is that the exposures of interest must be relevant to health. This requires consideration of the time-windows when damage occurs, as well as the routes of exposure, which contribute most to absorbed dose. The dermal route is an important, if not the most important, route of entry. Dermal exposure assessment techniques have been standardized and most regulatory agencies within the Organization for Economic Co-operation and Development (OECD) require whole-body dosimetric methods to be used. This may be useful for registration purposes, although this is not always relevant for epidemiological studies since the scientific basis for measuring whole-body dosimetry for a specific health effect is absent.

As a result, to date epidemiological studies of pesticide exposures have only been indicative of the presence of elevated health risks. Quantitative studies contributing to evidence on exposure-response relationships which could be used for quantitative risk assessment purposes are not widely available. This implies that the epidemiological potential has not been explored to its limits, as has been done for certain other agents such as asbestos and lead, for which present legislation has been based, to a large extent, on quantitative evidence of health risks in humans obtained from epidemiological studies.

It is often mentioned that observational epidemiological studies have many disadvantages, among which weaknesses in exposure assessment, and sensitivity to different forms of bias such as selection, information and confounding bias, are the major drawbacks (Nelson, 1988). Toxicological studies in animals with their greater control over the experimental design are sometimes considered superior. However, when available, good epidemiological studies are to be preferred when chemicals need to be classified or when quantitative risk assessments have to be performed. Why so few good epidemiological studies are available for pesticides 
is a matter of speculation. The fact that exposure assessment in epidemiology is a complex area, not always completely understood by epidemiologists, contributes to the explanation. Other factors might be the absence of technologies to evaluate the exposure to pesticides on a large scale in epidemiology studies, especially the absence of accurate techniques to evaluate dermal exposure and absence of insight in the most recent methodological developments. The principles of exposure assessment strategies for registration of pesticides and epidemiological studies differ. This seems not to have been completely understood by all researchers and so adds to the confusion. The fact that epidemiological studies on pesticides often have to be conducted in agricultural regions with the population spread over large areas, introducing logistic problems for quantitative exposure assessment strategies, contributes to the explanation for the absence of quantitative studies as well. However, even good or perfect epidemiological studies will not be able to answer all questions that need to be answered before risk assessments are possible. Epidemiological studies in agriculture, and even in pesticide-producing industries, may involve populations with exposure to mixtures of different pesticides. The conclusions that can be drawn from these studies are limited by this and usually do not allow specific conclusions about the particular risk associated with the use of one pesticide. Toxicological information will always be needed to complete the picture and give information about biological plausibility, and risk from individual agents. On the other hand, positive epidemiological findings from well-conducted studies, in the presence of negative toxicological findings, are usually a trigger for further studies. In addition, quantitative studies of exposures in occupationally and environmentally exposed populations are the only way to ascertain human exposure levels. This discussion illustrates that neither of the two scientific methods, epidemiology nor toxicology, give the complete answer.

Despite the fact that few epidemiological studies with quantitative exposure assessment data are available for pesticide exposure, more insight is now present on how the optimal exposure assessment strategy might look. In particular, the use of determinants of exposure studies, as reviewed recently, and their application in health-based exposure estimation, seems a promising approach that can solve many of the problems associated with pesticide exposure assessment in agriculture. This approach will be of use in both occupational and domestic epidemiological studies on this topic.

In summary, few epidemiological studies are currently available that have explored the limits with regard to quantitative exposure assessment to pesticides. It is also clear that exposure assessment in epidemiology is a complex process, but can be optimized by the application of existing principles and concepts. This will help improve the quality of epidemiological studies and allow the use of study results in quantitative risk assessment. A new generation of epidemiological pesticide studies is emerging. Such studies should help us develop a much more sophisticated understanding of the effects of pesticides in humans when proper exposure assessment strategies have been included. 


\section{REFERENCES}

Ahlborg, G.A. (1990). Validity of exposure data obtained by questionnaire, two examples from occupational reproductive studies, Scand. J. Work Environ. Health, 16, 284-288.

Arbuckle, T.E., R. Burnett, D. Cole, K. Teschke, M. Dosemecci, C. Bancej and J. Zhang (2002). Predictors of herbicide exposure in farm applicators, Int. Arch. Occup. Environ. Health, 75, 406-414.

Arbuckle, T.E., D. Cole, L. Ritter and B. Ripley (2004a). Farm Children's Exposure to Herbicides: Comparison of Biomonitoring and Questionnaire Data, Epidemiology, 15, 187-194.

Arbuckle, T.E., D. Cole, L. Ritter and B. Ripley (2004b). Biomonitoring of herbicides in Ontario farm applicators, Scand. J. Work Environ. Health (in press). ${ }^{1}$

Baumgarten, M., J. Siemiatycki and G.W. Gibbs (1983). Validity of work histories obtained by interview for epidemiologic purposes, Am. J. Epidem., 118, 583-591.

Blair, A. and S.H. Zahm (1990). Methodologic Issues in Exposure Assessment for CaseControl Studies of Cancer and Herbicides, Am. J. Ind. Med., 18, 285-293.

Blair, A. and S.H. Zahm (1993). Patterns of pesticide use among farmers: implications for epidemiologic research, Epidemiology, 4, 55-62.

Bohnen, N.I. and L.T. Kurland (1995). Brain Tumor and Exposure to Pesticides in Humans: A Review of the Epidemiologic Data, J. Neurolog. Sci., 132, 110-121.

Boleij, J.S.M., E. Buringh, D. Heederik and H. Kromhout (1995). Occupational Hygiene of Chemical and Biological Agents, Elsevier, Amsterdam, The Netherlands.

Bond, G.G., K.M. Bodner, W. Sobel, R.J. Shellenberger and G.H. Flores (1988). Validation of work histories obtained from interviews, Am. J. Epidem., 128, 343-351.

Bourbonnais, R., F. Meyer and G. Theriault (1988). Validity of self reported work history, Br. J. Ind. Med., 45, 29-32.

Bouyer, J., J. Dardenne and D. Hemon (1995). Performance of odds ratios obtained with a job-exposure matrix and individual exposure assessment with special reference to misclassification errors, Scand. J. Work Environ. Health, 21, 265-271.

Boyle, C.A., E.A. Brann and The Selected Cancers Co-operative Study Group (1992). Proxy Respondents and the Validity of Occupational and Other Exposure Data, Am. J. Epidem., 136, 712-721.

Brisson, C., M. Vezina, P.M. Bernard and S. Gingras (1991). Validity of occupational histories obtained by interview with female workers, Am. J. Ind. Med., 19, 523-530.

Brouwer, D.H., E.J. Brouwer and J.J. van Hemmen (1994). Estimation of long-term exposure to pesticides, Am. J. Ind. Med., 25, 573-88.

Brower, P.S. and M.D. Attfield (1998). Reliability of reported occupational history information for US Coal Miners, 1969 to 1977, Am. J. Epidem., 148, 920-926.

Burstyn, I. and K. Teschke (1999). Studying the determinants of exposure: a review of methods, Am. Ind. Hyg. Assoc. J., 60, 57-72.

Caldwell, G.G., S.B. Cannon, C.B. Pratt and R.D. Arthur (1981). Serum pesticide levels in patients with childhood colorectal carcinoma, Cancer, 48, 774-778.

Calvert, G.M., C.A. Mueller, V.L. O’Neill, J.M. Fajen, T. Briggle and L.E. Fleming (1997). Agreement between company recorded and self-reported estimates of duration and frequency of occupational fumigant exposure, Am. J. Ind. Med., 32, 364-368.

Checkoway, H., N.E. Pearce and D. Krübel (2004). Research Methods in Occupational Epidemiology, 2nd Edn, Oxford University Press, New York.

Ciccone, G. and P. Vineis (1988). Inter-rater agreement in the assessment of occupational exposure to herbicides, Med. lav., 79, 363-367.

Daniels, J.L., A.F. Olshan and D.A. Savitz (1997). Pesticides and Childhood Cancers, Environ. Health Perspect., 105, 1068-1077.

\footnotetext{
${ }^{1}$ As of August 2004.
} 
De Cock, J., K. Westveer, D. Heederik, E. te Velde and R. van Kooij (1994). Time to pregnancy and occupational exposure to pesticides in fruit growing in the Netherlands, Occup. Environ. Med., 51, 693-699.

De Cock, J., J. Kromhout, D. Heederik and J. Burema (1996). Experts' subjective assessment of pesticide exposure in fruit growing, Scand. J. Work Environ. Health, 22, $425-432$.

De Cock, J., D. Heederik, H. Kromhout, J.S.M. Boleij, F. Hoek, H. Wegh and E.T. Ny (1998a). Determinants of exposure to captan in fruit growing, Am. Ind. Hyg. Assoc. J., 59, $158-165$.

De Cock, J., D. Heederik, H. Kromhout, J.S.M. Boleij, F. Hoek, H. Wegh and E.T. Ny (1998b). Exposure to captan in fruit growing, Am. Ind. Hyg. Assoc. J., 59, 166-17.

Duares, J.P., I. Momas, J. Bernon and F. Gremy (1993). A vine-growing exposure matrix in the Hérault area of France, Int. J. Epidem., 22, S36-S41.

Eskenazi, B. and K. Pearson (1988). Validation of a self-administered questionnaire for assessing occupational and environmental exposures of pregnant women, Am. J. Epidem., 128, 1117-1129.

Flegal, K., C. Brownie and J. Haas (1986). The Effects of Exposure Misclassification on Estimates of Relative Risk, Am. J. Epidem., 123, 736-751.

Floderus, B., T. Persson, C. Stenlund, A. Wennberg, A. Ost and B. Knave (1993). Occupational exposure to electromagnetic fields in relation to leukemia and brain tumors: a case-control study in Sweden, Cancer Causes Control, 4, 465-476.

Hawkes, A.P. and J.R. Wilkins (1997). Assessing agreement between two job-exposure matrices, Scand. J. Work Environ. Health, 23, 140-148.

Heederik, D. and M. Attfield (2000). Characterization of dust exposure for the study of chronic occupational lung disease - a comparison of different exposure assessment strategies, Am. J. Epidem., 151, 982-990.

Hoar, S.K., A.S. Morrison, P. Cole and D.T. Silverman (1980). An occupation and exposure linkage system for the study of occupational carcinogenesis, J. Occup. Med., 22, $722-726$.

Hogenkamp, A., M. Vaal and D. Heederik (2004). Pesticide exposure in dwellings near bulb growing fields in The Netherlands: an explorative study, Ann. Agric. Environ. Med., 11, 149-153.

Hooiveld, M., D. Heederik, M. Kogevinas, P. Bofetta, L.L. Needham, D.G. Patterson and H.B. Bueno-de-Mesquita (1998). Second follow-up of a Dutch cohort occupationally exposed to phenoxy herbicides, chlorophenols, and contaminants, Am. J. Epidem., 147, 891-901.

Joffe, M. (1992). Validity of exposure data derived from a structured questionnaire, Am. J. Epidem., 135, 564-570.

Johnson, R.A., J.S. Mandel, R.W. Gibson, J.J. Mandel, A.P. Bender, P.D. Gunderson and C.M. Renier (1993). Data on prior pesticide use collected from self and proxy respondents, Epidemiology, 4, 157-164.

Kromhout, H., D. Heederik, L.M. Dalderup and D. Kromhout (1992). Performance of two general job-exposure matrices in a study of lung cancer morbidity in the Zutphen Cohort, Am. J. Epidem., 136, 698-711.

Kromhout, H., E. Symanski and S.M. Rappaport (1993). A comprehensive evaluation of within- and between-worker components of occupational exposure to chemical agents, Ann. Occup. Hyg., 37, 253-270.

Leighton, T.M. and A.P. Nielsen (1995). The United States Environmental Protection Agency, Health Canada and National Agricultural Chemicals Association pesticide handlers exposure database, Appl. Occup. Environ. Hyg., 10, 270-273.

Lerchen, M.L. and J.M. Samet (1986). An assessment of the validity of questionnaire responses provided by a surviving spouse, Am. J. Epidem., 123, 481-489. 
Linet, M.S., Stewart, W.F., Van Natta, M.L., McCaffrey, L.D. and Szklo, M. (1987). Comparison of methods for determining occupational exposure in a case-control interview study of chronic lymphocytic leukemia. J. Occup. Med., 29, 136-141.

Loewenhertz, C., Fenske, R.A., Simcox, N.J., Bellamy, G. and Kalman, D. (1997). Biological monitoring of organophosphorus pesticide exposure among children of agricultural workers in central Washington State, Environ. Health Perspect., 105, 1344-1353.

London, L. and J.E. Meyers (1998). Use of a crop and job specific exposure matrix for retrospective assessment of long term exposure in studies of chronic neurotoxic effects of agrichemicals, Occup. Environ. Med., 55, 194-201.

Loomis, D.P. and D.A. Savitz (1994). Effect of incomplete exposure assessment on epidemiologic dose-response analyses, Scand. J. Work. Environ. Health, 20, 200-205.

Maroni, M. and A. Fait (1993). Health Effects in Man From Long-Term Exposure to Pesticides - A Review of the 1975-1991 Literature, Toxicology, 78, 1-180.

McGuire, V., W.T Longstreth, L.M. Nelson, T.D. Koepsell, H. Checkoway, S. Morgan and G. van Belle (1997). Occupational Exposures and Amyotrophic Lateral Sclerosis. A Population-Based Case-Control Study, Am. J. Epidem., 145, 1076-1088.

McNamee, R. (1996). Retrospective assessment of occupational exposure to hydrocarbons-job-exposure matrices versus expert evaluation of questionnaires, Occup. Hyg., 3, 137-143.

Miligi, L., L. Settimi, G. Masala, P. Maiozzi, S. Alberghini Maltoni, A. Seniori Constantini, P. Vineis and The Working Group On Pesticide Exposure Assessment (1993). Pesticide Exposure Assessment: A Crop Exposure Matrix, Int. J. Epidem., 22(Suppl. 2), S42-S45.

Nanni, O., M. Ricci, C. Lugaresi, D. Amadori, F. Falcini and E. Buiatti (1993). Iterative use of a priori exposure matrices to improve the characterization of chemical exposures in agricultural work studies, Scand. J. Work Environ. Health, 19, 191-199.

Nelson, N. (1988). Toxicology and epidemiology: strengths and limitations, in Epidemiology and Health Risk Assessment, L. Gordis (Ed.), Oxford University Press, New York, pp. $5-13$.

Nieuwenhuijsen, M.J., K.S. Noderer and M.B. Schenker (1997). The relation between subjective dust exposure estimates and quantitative dust exposure measurements in California agriculture, Am. J. Ind. Med., 32, 355-363.

Pannett, B., D. Coggon and E.D. Acheson (1985). A job-exposure matrix for use in population based studies in England and Wales, Br. J. Ind. Med., 42, 777-783.

Pershagen, G. and O. Axelson (1982). A validation of questionnaire information on occupational exposure and smoking, Scand. J. Work Environ. Health, 8, 24-28.

Pickle, L.W., L.M. Brown and W.J. Blot (1983). Information available from surrogate respondents in case-control interview studies, Am. J. Epidem., 118, 99-108.

Preller, L., D. Heederik and H. Kromhout (1995). Determinants of dust and endotoxin exposure of pig farmers: development of a control strategy using empirical modeling, Ann. Occup. Hyg., 39, 545-557.

Rajan, B., R. Flesbury, B. Carton, M. Gerin, H. Litske, H. Marquart, E. Olsen, T. Scheffers, R. Stamm and T. Woldbaek (1997). European Proposal for Core Information for the Storage and Exchange of Workplace Exposure Measurements on Chemical Agents, Appl. Occup. Environ. Hyg., 12, 31-39.

Rappaport, S.M. (1991). Assessment of long-term exposures to toxic substances in air, Ann. Occup. Hyg., 35, 61-121.

Rocca, W.A., L. Fratiglioni, D.P. Bracco, C. Groppi and B.S. Schoenberg (1986). The use of surrogate respondents to obtain questionnaire data in case-control studies of neurologic diseases, J. Chronic Diseases, 39, 907-912.

Rodvall, Y., A. Ahlbom, B. Spannare and G. Nise (1996). Glioma and occupational exposure in Sweden, a case-control study, Occup. Environ. Med., 53, 526-532. 
Roeleveld, N., G.A. Zielhuis and F. Gabreels (1993). Mental retardation and parental occupation: a study on the applicability of job exposure matrices, Br. J. Ind. Med., 50, $945-954$.

Rona, R.J. and J. Mosbech (1989). Validity and repeatability of self-reported occupational and industrial history from patients in EEC countries, Int. J. Epidem., 18, 674-679.

Rosenberg, C.R., M.N. Mulvihill, A. Fischbein and S. Blum (1987). An analysis of the validity of self-reported occupational histories using a cohort of workers exposed to PCBs, Br. J. Ind. Med., 44, 702-710.

Rothman, K.J. and S. Greenland (1998). Modern Epidemiology, 2nd Edn, LippincottRaven, Philadelphia, PA, USA.

Rybicki, B.A., C. Cole Johnson, E.L. Peterson, G.X. Korsha and J.M. Gorell (1997). Comparability of different methods of retrospective exposure assessment of metals in manufacturing industries, Am. J. Ind. Med., 31, 36-43.

Scheele, J., M. Teufel and K.H. Niessen (1992). Chlorinated hydrocarbons in the bone marrow of children: studies on their association with leukemia, Eur. J. Pediatrics, 151, $802-805$.

Scheele, J., M. Teufel and K.H. Niessen (1996). Chlorinated hydrocarbons in the bone marrow of healthy individuals and leukemia patients, Arch. Environ. Health, 51, 22025.

Schneider, T., R. Vermeulen, D.H. Brouwer, J.W. Cherrie, H. Kromhout and C.L. Fogh (1999). Conceptual model for assessment of dermal exposure, Occup. Environ. Med., 56, 765-774.

Schnitzer, P.G., A.F. Olshan, D.A. Savitz and J.D. Erickson (1995). Validity of mother's report of father's occupation in a study of paternal occupation and congenital malformations, Am. J. Epidem., 141, 872-877.

Segnan, N., A. Ponti, G.F. Ronco, H. Kromhout, D. Heederik, J. De Cock, S. Bosia, L. Luccoli, P. Piccioni, A. Seniori Constantini, L. Miligi, A. Scarpelli, M. Mariotti, C. Scarnato and L. Morisi (1996). Comparison of Methods for Assessing the Probability of Exposure in Metal Plating, Shoe and Leather Goods Manufacture and Vine Growing, Occup. Hyg. 3, 199-208.

Sieber, W.K., D.S. Sundin, T.M. Frazier and C.F. Robinson (1991). Development, use and availability of a job exposure matrix based on national occupational hazard survey data, Am. J. Ind. Med., 20, 163-174.

Stewart, P. (1999). Exposure assessment in community-based epidemiological studies, Lancet, 353, 1816-1817.

Stewart, P.A. and W.F. Stewart (1994). Occupational Case-control studies: II. Recommendations for exposure assessment, Am. J. Ind. Med., 26, 313-326.

Stewart, W.F., J.A. Tonascia and G.M. Matanoski (1987). The validity of questionnairereported work history in live respondents, J. Occup. Med., 29, 795-800.

Stewart, P.A., W.F. Stewart, J. Siemiatycki, E.F. Heineman and D. Mustafa (1998). Questionnaires for collecting detailed occupational information for community-based case control studies, Am. Ind. Hyg. Assoc. J., 58, 39-44.

Teschke, K., C. Hertzman, H. Dimich-Ward, A. Ostry, J. Blair and R. Herschler (1989). A comparison of exposure estimates by worker raters and industrial hygienists, Scand. J. Work Environ. Health, 15, 424-429.

Teschke, K., S.M. Kennedy and A.F. Olshan (1994). Effect of different questionnaire formats on reporting of occupational exposures, Am. J. Ind. Med., 26, 327-337.

Tielemans, E., L. Kupper, H. Kromhout, D. Heederik and R. Houba (1998). Individualbased and group-based exposure assessment: some equations to evaluate different strategies, Ann. Occup. Hyg., 42, 115-119.

Tielemans, E., D. Heederik, A. Burdorf, R. Vermeulen, H. Veulemans, H. Kromhout and K. Hartog (1999a). Assessment of occupational exposures in a general population: comparison of different methods, Occup. Environ. Med., 56, 145-151. 
Tielemans, E., E. Louwerse, J. de Cock, D. Brouwer, D. Heederik and G. Zielhuis (1999b). Exposure to fungicides in fruit growing: re-entry time as a predictor for dermal exposure, Am. Ind. Hyg. Assoc. J., 60, 789-793.

van Der Gulden, J.W.J., I.W. Jansen, A.L.M. Verbeek and J.J. Kolk (1993). Repeatability of self-reported data on occupational exposure to particular compounds, Int. J. Epidem., 22, 284-287.

van Hemmen, J.J. (1992). Estimating Worker Exposure for Pesticide Registration, Rev. Environ. Contam. Toxicol., 128, 43-54.

Wang, F.L., K.M. Semchuk and E.J. Love (1994). Reliability of Environmental and Occupational Exposure Data Provided by Surrogate Respondents in a Case-Control Study of Parkinson's Disease, J. Clin. Epidem., 47, 797-807.

Wärneryd, B., M. Thorslund and P. Ostlin (1991). The quality of retrospective questions about occupational history - a comparison between survey and census data, Scand. $J$. Soc. Med., 19, 7-13.

Zahm, S.H. and A. Blair (1993). Cancer Among Migrant and Seasonal Farmworkers: An Epidemiologic Review and Research Agenda, Am. J. Ind. Med., 24, 753-766.

Zahm, S.H. and M.H. Ward (1998). Pesticides and Childhood Cancer, Environ. Health Perspect., 106, 893-908. 